\title{
Mutations in FOXC2 in Humans (Lymphoedema Distichiasis Syndrome) Cause Lymphatic Dysfunction on Dependency
}

\author{
Russell H. Mellor ${ }^{a}$ Naomi Tate ${ }^{a}$ Anthony W.B. Stanton ${ }^{a}$ Charlotte Hubert $^{a}$ \\ Taija Mäkinen $^{d}$ Alberto Smith $^{\mathrm{e}}$ Kevin G. Burnand ${ }^{\mathrm{e}}$ Steve Jeffery ${ }^{\mathrm{b}}$ \\ J. Rodney Levick ${ }^{c}$ Peter S. Mortimer ${ }^{a}$ \\ ${ }^{a}$ Cardiac and Vascular Sciences (Dermatology), ${ }^{b}$ Clinical Developmental Sciences (Medical Genetics), 'Basic Medical \\ Sciences (Physiology), St. George's, University of London, ' Lymphatic Development Laboratory, Cancer Research \\ UK London Research Institute, and 'Academic Surgery, St. Thomas' Hospital, London, UK
}

\section{Key Words}

Dependent oedema • Fluorescence microlymphography •

Lymphatic vessels $\cdot$ Lymphoedema $\cdot$ Skin

\begin{abstract}
Background: Human lymphoedema distichiasis syndrome (LDS) results from germline mutations in transcription factor FOXC2. In a mouse model, lack of lymphatic and venous valves is observed plus abnormal smooth muscle cell recruitment to initial lymphatics. We investigated the mechanism of lymphoedema in humans with FOXC2 mutations, specifically the effect of gravitational forces on dermal lymphatic function. Methods: We performed (1) quantitative fluorescence microlymphangiography (FML) on the skin of the forearm (non-swollen region) at heart level, and the foot (swollen region) below heart level (dependent) and then at heart level, and (2) immunohistochemical staining of microlymphatics in forearm and foot skin biopsies, using antibodies to podoplanin, LYVE-1 and smooth muscle actin. Results: FML revealed a marked reduction in fluid uptake by initial lymphatics in the LDS foot during dependency, yet normal uptake (similar to controls) in the same foot at heart level and in LDS forearms. In control subjects, dependency did not impair initial lymphatic filling. Immunohistochemical micro-
\end{abstract}

lymphatic density in forearm and foot did not differ between LDS and controls. Conclusions: FOXC2 mutations cause a functional failure of dermal initial lymphatics during gravitational stress (dependency), but not hypoplasia. The results reveal a pathophysiological mechanism contributing to swelling in LDS.

Copyright $\odot 2011$ S. Karger AG, Basel

\section{Introduction}

Lymphoedema distichiasis syndrome (LDS) (fig. 1) is an inherited primary lymphoedema with causative mutations in the FOXC2 gene [1-4], a forkhead transcription factor gene implicated in lymphatic development $[5,6]$. LDS is characterised by bilateral lower limb lymphoedema (usually asymmetrical) presenting at or after puberty, plus an abnormal second row of eye lashes developing from a dysfunctional meibomian gland. The distichiasis, unlike the lymphoedema, is present from birth [7]. The delayed onset of the swelling contrasts with the other he-

R.H.M., A.S., K.G.B, S.J. and P.S.M. are members of the Lymphoedema Research Consortium.

\section{KARGER}

Fax +41613061234 E-Mail karger@karger.ch www.karger.com

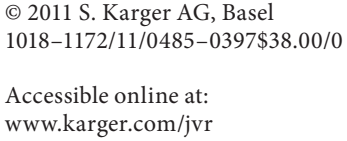

Prof. Peter S. Mortimer

Cardiac and Vascular Sciences (Dermatology)

St. George's, University of London

Cranmer Terrace, London SW17 0RE (UK)

Tel. +44 208725 1784, Fax +44208725 5353, E-Mail mortimer@sgul.ac.uk 

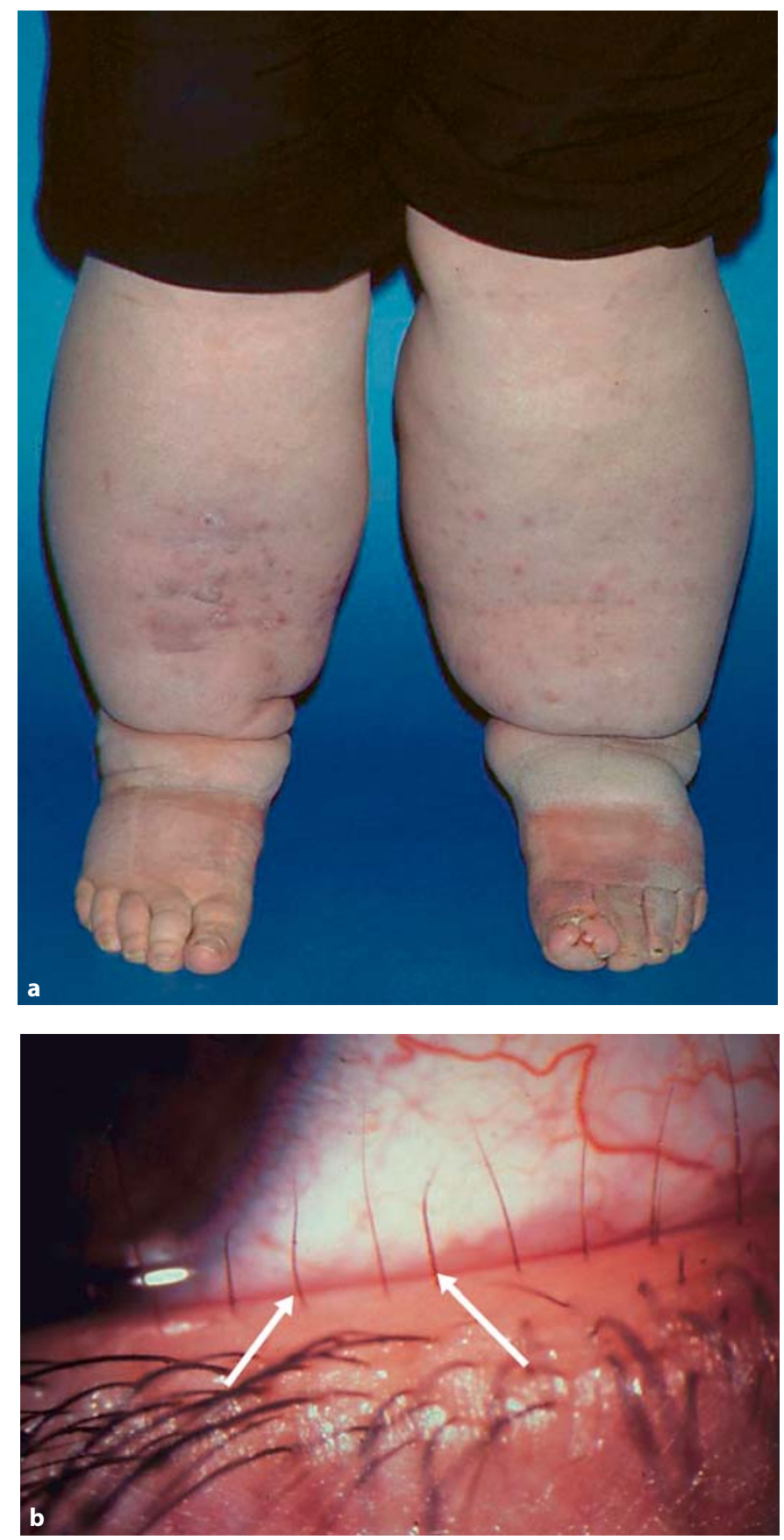

Fig. 1. Clinical manifestations of LDS. a Bilateral leg lymphoedema. b Distichiasis (double row of eye-lashes). The extra row of eyelashes along the posterior border of the lid margin is arrowed.

reditary lymphoedemas with causal mutations, Milroy disease, hypotrichosis-lymphoedema-telangiectasia and generalised lymphatic dysplasia, in which the swelling is present from birth [8-10]. The germline mutation in LDS is by definition present in all cells but the swelling is con- fined to the legs and feet. It is unclear why these tissues swell whilst the rest of the body does not.

Few studies have explored the mechanism for the oedema in LDS. X-ray lymphangiography in 5 cases of primary lymphoedema with distichiasis (from a total of 725 reviewed cases of primary lymphoedema) reportedly showed bilateral hyperplasia, but no further description or images were provided [11]. Lymphangiography in 5 LDS patients known to have mutations in FOXC2 showed increased imaging of ilio-inguinal lymph nodes, with the number of leg lymph channels at the upper limit of normal [7]. Lymphoscintigraphy following subcutaneous injection of radiocolloid in the feet in LDS showed extensive re-routing of tracer throughout the lower limbs, most marked below the knees (characteristic dermal backflow) and considered to indicate lymph reflux [7]. Functional assessment revealed abnormally low uptake of radioactive colloid in the ilio-inguinal nodes at 30 and $60 \mathrm{~min}$ in 9 of 11 patients; the patients would have been ambulant in the interval between imaging in the supine position.

Normal lymphatic collector vessels possess semilunar luminal valves, similar to those in veins, which prevent retrograde lymph flow. Foxc $2^{-1-}$ mice lacked lymphatic valves allowing retrograde filling of lymphatics following injection of fluorescent marker. They also exhibited an abnormal mural investment of smooth muscle actin (SMA)-positive peri-endothelial cells in the dermal lymphatic capillaries, which were also ectatic [12]. Lymphatic smooth muscle cells are normally restricted to larger lymphatic vessels, where their contractile activity propels lymph centrally. The Foxc2 $2^{-/-}$mouse model raises the possibility that a dysfunctional interaction between initial lymphatic endothelial cells and abnormal mural cells and/or defects in the more proximal lymphatic semilunar valves might be the cause of the swelling in LDS. It is not known, however, whether either or both defects occur in humans with mutations in FOXC2. Based on the embryological link between veins and lymphatics, we recently tested the hypothesis that venous valve function may be impaired in LDS, venous valve function being considerably easier to test than lymphatic valve function. Colour duplex ultrasound revealed multiple venous valve failure in the legs of participants with FOXC2 mutations; 100\% of participants had great saphenous (superficial) vein reflux and $78 \%$ had deep vein reflux [13]. We concluded that functioning FOXC2 is required for normal venous function, and more specifically for venous valve development and/or maintenance in humans.

Venous pressure is normally $75-85 \mathrm{~mm} \mathrm{Hg}$ higher in the feet than at heart level during stationary orthostasis. 
The resulting increase in capillary hydrostatic pressure raises the rate of fluid filtration into the interstitium of the feet [14]. This in turn raises the interstitial hydrostatic pressure and reduces the interstitial colloid osmotic pressure [15]. Both these interstitial changes act to limit the filtration rate; even so, foot swelling is mainly prevented in the healthy individual by an increase in the rate of drainage of interstitial fluid into the initial lymphatic network, increasing the leg lymph flow [16]. Lymph flow is coupled to interstitial matrix hydration and during gravitational stress (dependency) there is an increase in the contractile activity of the major lymphatics in the leg (chiefly the subcutaneous collector vessels), which drives the increased lymph load proximally $[17,18]$. With venous valve dysfunction, venous pressure is chronically raised distally and hence also the microvascular filtration pressure, leading to an increased capillary filtration rate in the dependent legs and feet as well as a greater fluid load on the lymphatic system. A defect in the lymphatic semilunar valves would greatly exacerbate the effect of gravity on leg/foot microvascular filtration (with or without venous valve dysfunction) and promote swelling. The action of gravity on an uninterrupted vertical column of fluid (lymph) would increase distal lymph pressure and thus oppose fluid drainage from the interstitium into the initial lymphatic plexus.

We hypothesised, therefore, that the effect of gravity on the lymphatic system is affected by a FOXC2 germline mutation such as to impair interstitial fluid uptake into initial lymphatic system in the dependent leg. To test this hypothesis we performed intravital fluorescence microlymphangiography (FML) on the skin of the swollen foot, both at heart level and during dependency, and also in the non-swollen forearm. FML is a semi-quantitative method that tests the ability of the lymphatic capillary network to take up a dyed fluid from the interstitial compartment. The following questions were addressed in LDS patients. (2) Are functioning initial lymphatics (irrespective of their mutation status) present in the skin? (2) If functioning initial lymphatics are indeed present in the skin of the foot, is filling affected by gravitational stress (dependency)? (3) If functioning initial lymphatics (that is, lymphatics that take up dyed fluid during FML) are depleted or absent in the swollen foot, can lymphatics nevertheless be demonstrated histologically, that is, is the problem one of lymphatic function or lymphatic numbers? To answer this last question, we used immunocytochemistry of skin biopsies to quantify the histological density of the initial lymphatics, and any schism between histology and function was thus assessed.

FOXC2 Mutations Cause Lymphatic Dysfunction

\section{Methods}

Three groups were examined.

LDS group (penetrant FOXC2 group): 12 participants (8 male, 4 female) aged $48.7 \pm 11.8$ years (mean \pm standard deviation, range 21-61 years), all with known mutations in FOXC2 (small deletions or insertions that induce a frameshift), were recruited from 8 families. All had bilateral lymphoedema affecting the feet and distichiasis. One did not undergo skin biopsy.

Control group: this comprised 12 participants ( 6 male, 6 female) aged $38.0 \pm 11.7$ years (23-56 years) with no mutation in FOXC2, no lymphoedema and no distichiasis on examination. Eight were family members of the LDS group and 4 were unrelated. One did not undergo skin biopsy.

FOXC2 carrier group (non-penetrant FOXC2 group): 3 participants (1 male, 2 female) aged 20, 31 and 36 years, with mutations in FOXC2, were examined by FML only (they declined skin biopsy). None had lymphoedema on examination.

The study was approved by the National Research Ethics Service (Wandsworth and St. Thomas' Hospital Protocol No. 03.0015 and EC03/139) and each participant gave informed, written consent.

Intravital Fluorescence Microlymphangiography

The procedure, adapted from that of Bollinger et al. [19], has been used extensively in our laboratory [20-22]. Three skin sites were examined in turn in each individual.

(1) The foot with the worse swelling was selected for study by FML in the LDS group, and either foot was selected in the other two groups. The participant was seated, with the legs dependent, for $60 \mathrm{~min}$ beforehand. The dorsum of the foot was examined in the dependent position with the participant reclining at approximately $45^{\circ}$. The vertical distance of the foot below the mid-right atrial level was similar in the LDS $(80.4 \pm 8.0 \mathrm{~cm})$ and control groups $(76.6 \pm 5.3 \mathrm{~cm}, \mathrm{p}=0.20$, Student's unpaired t test); the distances were not precisely identical because of height differences in the groups. The mid-right atrial level ('heart level') was taken to be $5-6 \mathrm{~cm}$ below the manubriosternal angle.

(2) The ventral forearm (a non-swollen region in the LDS group) was examined at heart level by FML following a 4-hour period spent away from the laboratory plus $30 \mathrm{~min}$ acclimatisation (seated).

(3) The dorsum of the same foot was then re-examined by FML when positioned at heart level. For this examination the participant was supine and had been lying down for $45 \mathrm{~min}$ prior to dye injection.

The two FML injections (see below) on the same foot were thus well separated in time (by $5.5 \mathrm{~h}$ ) and also by distance (by $5 \mathrm{~cm}$ between the injection sites), so that lymphatic filling during the first, dependent study did not influence the subsequent study at heart level.

Fluorescein isothiocyanate-dextran ( $5 \mu \mathrm{l}$ of $25 \%$ w/v solution, dextran molecular mass approx. $150 \mathrm{kDa}$; Sigma, Poole, UK) was injected intradermally over approximately $10 \mathrm{~s}$ using a 36 gauge needle of outer diameter $0.2 \mathrm{~mm}$ (Unimed, Zurich, Switzerland) and a glass Hamilton 705 LT $50 \mu$ l syringe (ESSLAB, Hadleigh, UK). The network of initial lymphatics (lymphatic capillaries) around the fluorescein isothiocyanate -dextran depot gradually filled with fluorescent tracer. The network and depot were viewed using a fluorescence videomicroscope (modified DMLB; Leica, 
Milton Keynes, UK) and recorded onto videotape for $45 \mathrm{~min}$ after the injection. Prints were made direct from the videotape with a final magnification of $\times 35.5$.

\section{Analysis of Lymphangiograms}

A lymphangiogram was produced from a montage of videoprints, using the clearest images obtained for each site examined by FML. All the lymphatic vessels that took up tracer over the 45 min recording period were assembled in the lymphangiogram. A skeletal map was then traced over the lymphangiogram, each lymphatic being depicted as a solid black line. Measurements were made from the skeletal map by both stereological analysis and directly, as described previously [20-22]. The measurements, in brief, were as follows.

\section{Stereological Measurements}

A grid of concentric circles with radii increasing in 1-cm steps was drawn on a transparent sheet (equivalent to $282-\mu \mathrm{m}$ steps on the skin), extending from the depot centre to the furthermost filled lymphatic. The grid was placed over the skeletal lymphangiogram. Lymphatic length density (units: $\mathrm{cm} / \mathrm{cm}^{2}, \mathrm{~cm}^{-1}$ ) was determined for each circle by the line intersection method, using the stereological formula: lymphatic density $=N \pi / 2 C$, where $N$ is the number of intersections of lymphatics with the grid line and $\mathrm{C}$ is the circumference of the grid line [23]. This procedure generated a set of filled lymphatic densities at increasing radial distances from the depot (radial lymphatic density, $\mathrm{LD}_{\mathrm{r}}$ ) for each lymphangiogram. Peak $\mathrm{LD}_{\mathrm{r}}$ was defined as the highest individual $\mathrm{LD}_{\mathrm{r}}$ in the set; this was usually located close to the depot. A further parameter, termed maximum lymphatic length density $\left(\mathrm{LD}_{\max }\right)$, was obtained as follows. The region of greatest lymphatic filling was selected by inspection and a grid of 3 concentric circles of radii $1-3 \mathrm{~cm}$ (with an overall area equivalent to $25 \mathrm{~mm}^{2}$ on the skin) was placed over this region. The line intersection method was used to calculate density for each circle, as above, and the average taken.

Derived and Non-Stereological Measurements

(1) Total lymphatic length (LL, cm) was defined as the total length of tracer-filled lymphatics. Vessel length in each individual annulus of the grid was calculated as local $\mathrm{LD}_{\mathrm{r}}$ multiplied by the area of the associated $282-\mu \mathrm{m}$-wide annulus. LL was calculated as the sum of the vessel lengths in all the annuli.

(2) Maximal spread $(\mathrm{cm})$ was defined as the radial distance from the centre of the depot to the furthermost tracer-filled lymphatic vessel.

(3) Percent sector filled: the filling of the network around the depot is often patchy. The percent sector filled was defined as the angular sum of the sectors around the depot containing tracerfilled lymphatics, expressed as a percentage of $360^{\circ}$.

\section{Skin Biopsies and Lymphatic Immunohistochemistry}

Procedures were as described previously [24]. Podoplanin and LYVE-1 immunohistochemistry gave similar results in a recent study of Milroy disease but differed in absolute vessel counts; LYVE-1 stains both lymphatic endothelial cells and macrophages, and can give misleadingly high counts, whereas podoplanin, used here for quantification, is probably more selective for lymphatic endothelial cells [24]. Briefly, a 6-mm punch biopsy (Stiefel biopsy punch; Medisave, Weymouth, UK) was taken from the skin of the dorsal foot and ventral forearm of 9 FOXC2+/- patients and 10 controls, following local intradermal administration of $2 \%$ lidocaine without adrenaline (Kent Pharmaceuticals, Ashford, UK). Biopsies were fixed immediately in $10 \%$ formalin and subsequently embedded in paraffin. For quantification, a rat monoclonal antibody to human podoplanin (NZ-1; Angiobio, Del Mar, Calif., USA) $[25,26]$ was used as a marker for lymphatic vessels on 5 - $\mu \mathrm{m}$ serial sections after heat-induced epitope retrieval. The sections were then stained with Cy2-conjugated donkey anti-rat antibodies (secondary fluorescent antibody) (Jackson ImmunoResearch, West Grove, Pa., USA). In order to clearly distinguish the initial lymphatics from the blood vessels on each section the blood vessels were identified using Cy3-conjugated mouse antibody against $\alpha$-SMA (Sigma). The blood vessels were not counted. A rabbit polyclonal antibody to LYVE-1 (Reliatech, Wolfenbüttel, Germany) was used as a second marker of (initial) lymphatic vessels. The slides were viewed and images were captured using Zeiss Axiobserver Z1 microscope equipped with a monochrome Hamamatsu Orca-AG camera and MetaMorph software (Molecular Devices, Sunnyvale, Calif., USA).

\section{Image Acquisition and Quantitative Analysis of Stained}

Sections

Sections were viewed at $\times 200$ and greyscale images captured with a microscope-mounted CoolSnap-Pro CF colour digital camera (Leitz DMRB; Leica, and Media Cybernetics, Bethesda, Md., USA) and ProScan motorised stage (Prior Scientific, Cambridge, UK). Multiple images were compiled into a single composite image of the whole skin section, using image analysis software (Image-Pro Plus; Media Cybernetics). One complete image was analysed per biopsy. A vessel was counted as a lymphatic if it was podoplanin positive and had a lumen. Lymphatic vessel density was calculated as the total number of lymphatic vessels per unit cross-sectional area of the composite image of dermis.

Statistical Analysis

Analysis was performed using GraphPad Prism (version 4.03; GraphPad, La Jolla, Calif., USA). Means \pm SD are given in the text and table, and means \pm SEM are shown in the figures. Statistical comparisons were performed using Student's paired and unpaired $t$ tests and, for multiple comparisons, two-way analysis of variance (ANOVA) with the Bonferroni post-test. Differences were considered significant if $\mathrm{p}<0.05$.

\section{Results}

\section{Fluorescence Microlymphangiography}

Forearm versus Foot at Heart Level (All Groups)

The initial lymphatic vessel network was denser and more extensive in the foot at heart level than in the forearm in both the control and penetrant $\mathrm{FOXC} 2+/$ - groups (fig. 2, 3; table 1). This finding mirrored our previous report that filled lymphatic density is greater in the leg (in the skin covering the shin) than forearm in healthy humans [22]. 
Fig. 2. Joined plots of functional lymphatic measurements from the dermis of the ventral forearm at heart level (HL), the dorsal foot at heart level, and the dorsal foot during dependency in the healthy control and lymphoedema distichiasis groups. Results from 12 participants for each group undergoing intravital FML. a Total lymphatic length (LL). b Maximum lymphatic density $\left(\mathrm{LD}_{\max }\right)$.
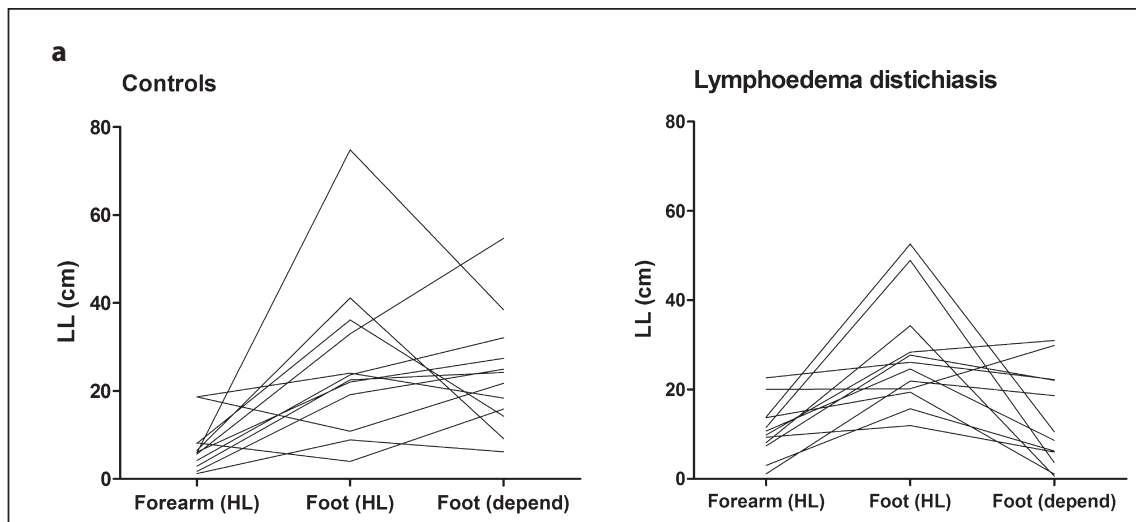

b
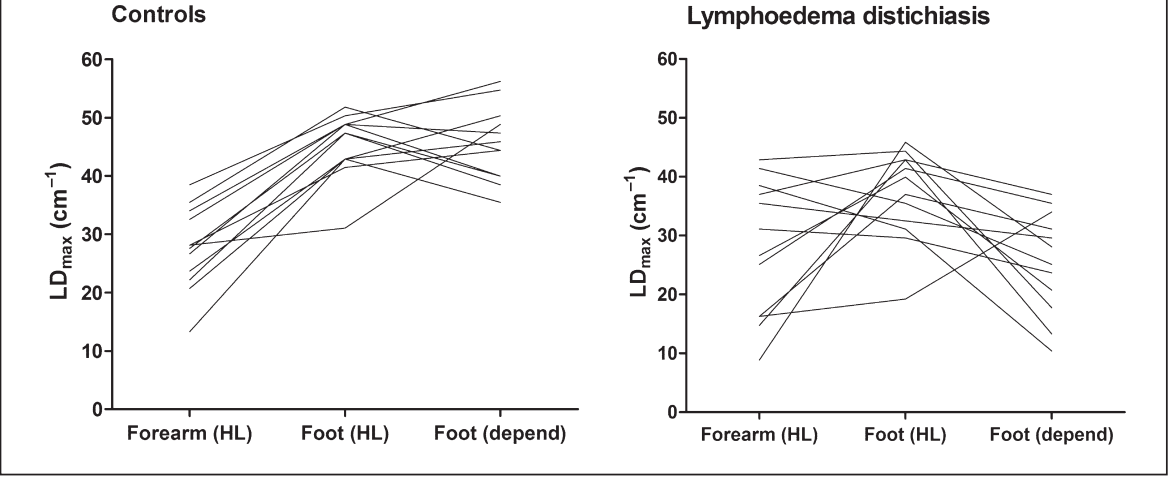

Table 1. Quantification of lymphatic filling

\begin{tabular}{|c|c|c|c|c|c|c|c|c|}
\hline & \multicolumn{3}{|c|}{ Controls $(\mathrm{n}=12)$} & \multirow[t]{2}{*}{$\mathrm{p}^{\mathrm{a}}$} & \multicolumn{3}{|c|}{ Lymphoedema distichiasis $(\mathrm{n}=12)$} & \multirow[t]{2}{*}{$\mathrm{p}^{\mathrm{a}}$} \\
\hline & forearm $(\mathrm{HL})$ & foot $(\mathrm{HL})$ & foot (depend) & & forearm (HL) & foot (HL) & foot (depend) & \\
\hline $\mathrm{LL}, \mathrm{cm}$ & $7.3 \pm 5.8$ & $26.7 \pm 18.7$ & $23.9 \pm 13.4$ & 0.62 & $10.9 \pm 6.2$ & $27.6 \pm 12.3$ & $13.4 \pm 10.9$ & 0.017 \\
\hline $\mathrm{LD}_{\max }, \mathrm{cm}^{-1}$ & $27.6 \pm 7.0$ & $45.4 \pm 5.6$ & $45.5 \pm 6.4$ & 0.96 & $27.9 \pm 11.6$ & $36.9 \pm 7.7$ & $25.5 \pm 8.7$ & 0.008 \\
\hline Peak LD, $\mathrm{cm}^{-1}$ & $16.2 \pm 7.4$ & $27.1 \pm 10.6$ & $29.0 \pm 10.1$ & 0.56 & $19.4 \pm 8.3$ & $23.6 \pm 6.2$ & $17.7 \pm 7.0$ & 0.029 \\
\hline Max. spread, cm & $0.8 \pm 0.2$ & $1.4 \pm 0.7$ & $1.4 \pm 0.5$ & 0.80 & $1.3 \pm 0.8$ & $1.9 \pm 0.62$ & $1.3 \pm 0.8$ & 0.11 \\
\hline$\%$ sector filled & $65.7 \pm 22.7$ & $83.2 \pm 17.3$ & $85.1 \pm 17.7$ & 0.73 & $75.5 \pm 23.9$ & $87.2 \pm 11.6$ & $71.7 \pm 26.1$ & 0.06 \\
\hline
\end{tabular}

Quantification of lymphatic filling at heart level (HL) and during dependency (depend) by intravital fluorescence microlymphangiography of the forearm and foot in the healthy control and lymphoedema distichiasis groups (means \pm SD).

a Student's paired t test comparing the foot at heart level with the dependent foot.

In the control group LL was $5.6 \pm 3.9$ times greater in the foot at heart level than in the forearm, while in the FOXC2+/- group it was $4.2 \pm 4.9$ times greater (fig. 3; $\mathrm{p}=0.51$ for controls vs. FOXC2+/-, $\mathrm{n}=12$ for each group, two-way ANOVA with Bonferroni post-test). $\mathrm{LD}_{\max }$ was $1.8 \pm 0.6$ times greater in the foot at heart level than forearm in the controls, with a similar difference in the FOXC2+/- group (1.7 \pm 1.3 times) (fig. $3 ; \mathrm{p}=0.12$ for con- trols vs. FOXC2+/-). Peak $\mathrm{LD}_{\mathrm{r}}$ showed the same pattern, being $2.5 \pm 2.9$ times greater in the foot at heart level than forearm in the controls and $1.5 \pm 1.0$ times greater in the FOXC2+/- group ( $\mathrm{p}=0.97$ for controls vs. FOXC2+/-). The maximal spread and percent sector filled findings were consistent with the above but did not achieve significance at the conventional level (table 1). The two-way ANOVA and post-test results presented 


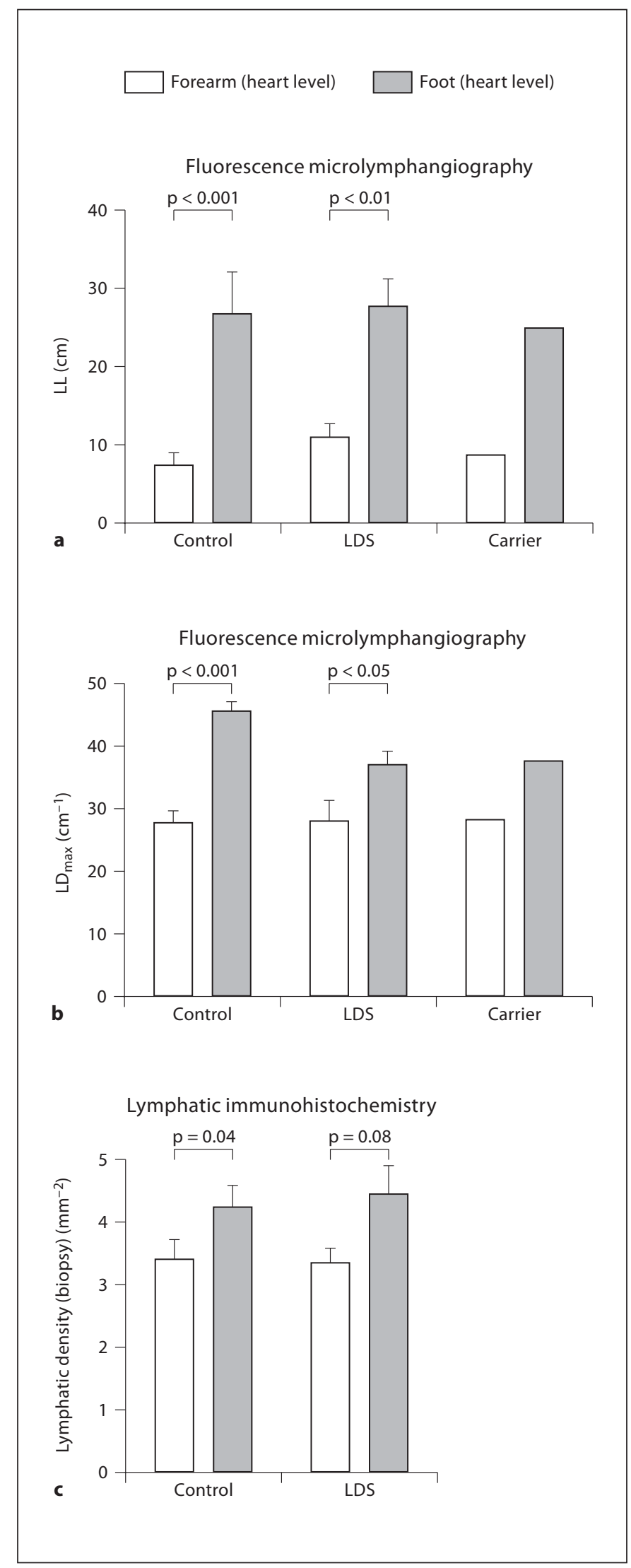

above showed that lymphatic filling did not differ significantly between the control and LDS groups, when both tissues were at heart level.

In the non-penetrant FOXC2 carrier group $(\mathrm{n}=3)$ lymphatic filling was likewise greater in the foot at heart level than the forearm (by 1.2-3.4 times). The pattern of FML findings in the carriers was thus similar to that in control and FOXC2 penetrant groups (fig. 3).

Foot at Heart Level versus Dependent Foot

The dependent foot showed a much reduced degree of lymphatic filling in the penetrant FOXC2+/- group, but not in the control group (table 1; fig. 2, 4). In the control group, dependency caused no statistically significant change in any of the five lymphatic uptake measurements in the foot ( $\mathrm{p}>0.5, \mathrm{n}=12$, Student's paired $\mathrm{t}$ tests), though the numerical trend was towards increased lymphatic filling (by 2-27\%) (fig. 4).

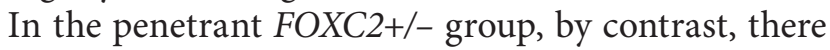
was a marked and statistically significant reduction in lymphatic filling in the foot during dependency for all five lymphatic measurements (fig. 4). LL fell by $44 \pm 46 \%$ ( $\mathrm{p}=0.017, \mathrm{n}=12$, Student's paired t tests), $\mathrm{LD}_{\max }$ fell by $26 \pm 39 \%(\mathrm{p}=0.008)$ and peak $\mathrm{LD}_{\mathrm{r}}$ fell by $21 \pm 36 \%$ $(\mathrm{p}=0.029)$. The fall in maximal spread, by $21 \pm 56 \%$ $(\mathrm{p}=0.11)$ and the $\%$ sector filled $(17 \pm 9 \%, \mathrm{p}=0.06)$ did not reach the conventional significance level.

In the non-penetrant FOXC2 carrier group $(\mathrm{n}=3)$, the change in lymphatic filling on dependency was -9 to $(+) 31 \%$, resembling the pattern of lymphatic filling seen in the controls (fig. 4).

\section{Lymphatic Immunohistochemistry}

Cutaneous lymphatic vessels were present in all sections in the FOXC2+/- group, in both foot and forearm (fig. 5). As previously reported [12], abnormal smooth muscle cell coverage of initial lymphatic vessels was observed in the foot of the FOXC2+/- group. In addition, the initial lymphatics showed lower LYVE-1 immunoreactiv-

Fig. 3. Functional and histological lymphatic density in the dermis of the ventral forearm and dorsal foot at heart level in controls and lymphoedema distichiasis. a Total lymphatic length (LL) measured by intravital FML. b Maximum lymphatic density $\left(\mathrm{LD}_{\max }\right)$ measured by intravital FML. c Lymphatic density determined by immunohistochemical staining of biopsies. $p$ value for $\mathrm{LL}$ and $\mathrm{LD}_{\max }$, Bonferroni post-test; $\mathrm{p}$ value for histological density, Student's paired t test. The foot has a higher functional and histological lymphatic density than the forearm. 
ity when compared to the control. The initial lymphatics of the FOXC2+/- arm were normal, however, indicating that ectopic smooth muscle cell recruitment onto the lymphatic vessels may occur secondarily for reasons other than germline mutation. The lymphatic densities determined by immunohistochemistry were similar in the control and FOXC2+/- subjects for the same site. As with the FML measurements, lymphatic density was markedly higher in the foot than forearm (fig. 3). In the control group the histological lymphatic density in the foot, 4.24 $\pm 1.12 \mathrm{~mm}^{-2}$, was $1.31 \pm 0.41$ times greater than in the forearm, which was $3.40 \pm 1.03 \mathrm{~mm}^{-2}(\mathrm{n}=10)$. Similarly, in the FOXC2+/- group the histological lymphatic density in the foot, $4.45 \pm 1.39 \mathrm{~mm}^{-2}$, was $1.39 \pm 0.58$ times greater than in the forearm $(3.35 \pm 0.70, \mathrm{n}=9)(\mathrm{p}=0.010$ for forearm vs. foot, $\mathrm{p}=0.83$ for control vs. LDS groups, two-way ANOVA). These observations show that the difference between lymphatic filling in the forearm and foot at heart level observed by FML has, at least in part, an anatomical basis.

\section{Discussion}

The principal findings were as follows. (1) Placing the foot in dependency substantially reduced the filling of initial lymphatics with tracer in the foot of the penetrant FOXC2+/- group, but had no statistically significant effect in the controls. (2) Despite a germline mutation of FOXC2 no functional or histological abnormalities of dermal lymphatics (neither failure of dye uptake nor reduced density nor abnormal smooth muscle investment of initial lymphatics) was found in forearm skin (which was non-swollen). (3) With both sites at heart level there was a consistently greater density and extent of tracerfilled lymphatics in the foot than in the forearm, in both the control and FOXC2+/- groups. (4) Immunohistochemistry confirmed that the density of dermal microlymphatics was higher in the foot than forearm in the control and FOXC2+/- groups, though the difference was less marked than that observed for functioning lymphatics identified by FML. (5) In the non-penetrant FOXC2 carriers without oedema the filling of the lymphatics showed no marked change in response to dependency, as in the controls.

The results indicate, therefore, that the initial lymphatic plexus of the skin of the swollen foot responded abnormally when challenged by dependency below heart level; fewer lymphatics showed fluid uptake than at heart level. The genetic mutation in LDS, present in all cells, did

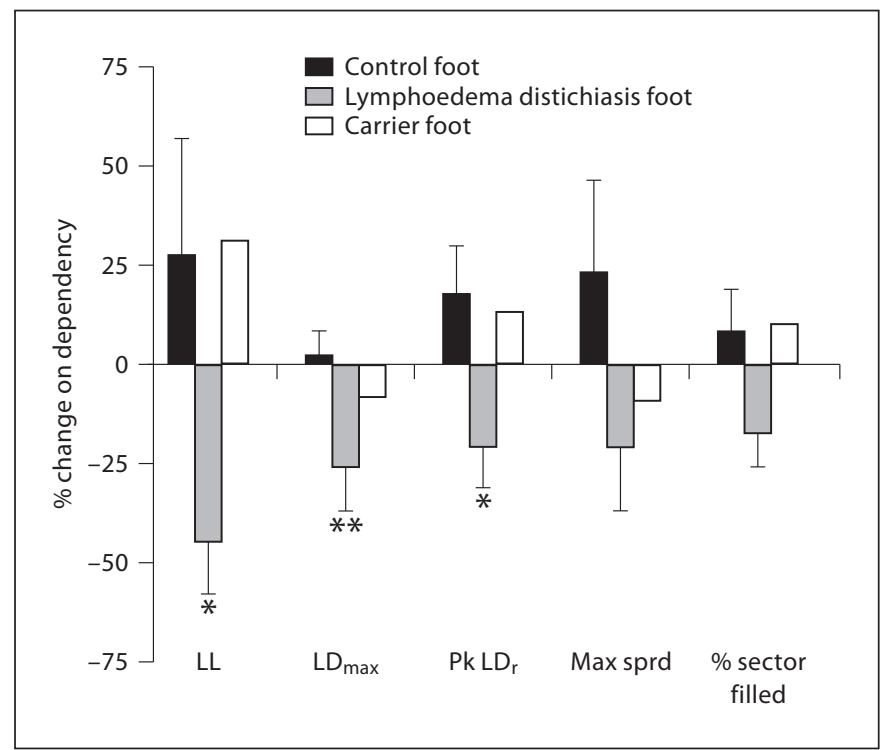

Fig. 4. Percentage change in functional lymphatic density and spread in the foot during dependency relative to measurements from the foot positioned at heart level. Mean + SEM from participants undergoing intravital FML. In the lymphoedema distichiasis group $(n=12)$ all measurements of lymphatic function fell during dependency, whereas in the healthy controls $(n=12)$ and carriers $(n=3)$ the measurements changed little or increased nonsignificantly. $\mathrm{LL}=$ Total lymphatic length; $\mathrm{LD}_{\max }=$ maximum lymphatic density; $\mathrm{Pk} \mathrm{LD}_{\mathrm{r}}=$ peak radial lymphatic density; Max sprd $=$ maximal spread. ${ }^{*} \mathrm{p}<0.05 ;{ }^{* *} \mathrm{p}<0.01$, Student's paired $\mathrm{t}$ tests. For explanation of lymphatic measurements, see text.

not influence the density and extent of functioning lymphatics in the forearm, a region closer to heart level than the foot during normal use and hence less affected by gravitational loading.

\section{Response to Dependency in Health}

Lymphatic function normally adapts to an increase in microvascular filtration rate so as to prevent or minimise oedema formation [27]. During orthostasis gravity causes a rise in microvascular filtration pressure in the dependent tissues such as the foot [14]. The resulting increase in microvascular filtration into the feet and legs is usually matched by an increase in lymph flow through the large trunk lymphatics of the legs, especially in ambulatory humans $[17,18,27,28]$. In keeping with this, the density and extent of the dye-filled initial lymphatic network is much greater in the skin over the shin than on the ventral forearm (both examined at heart level) in healthy young adult men, indicating a greater capacity to transport interstitial fluid into the initial lymphatics in the leg 
Podoplanin $\alpha$ SMA LYVE-1
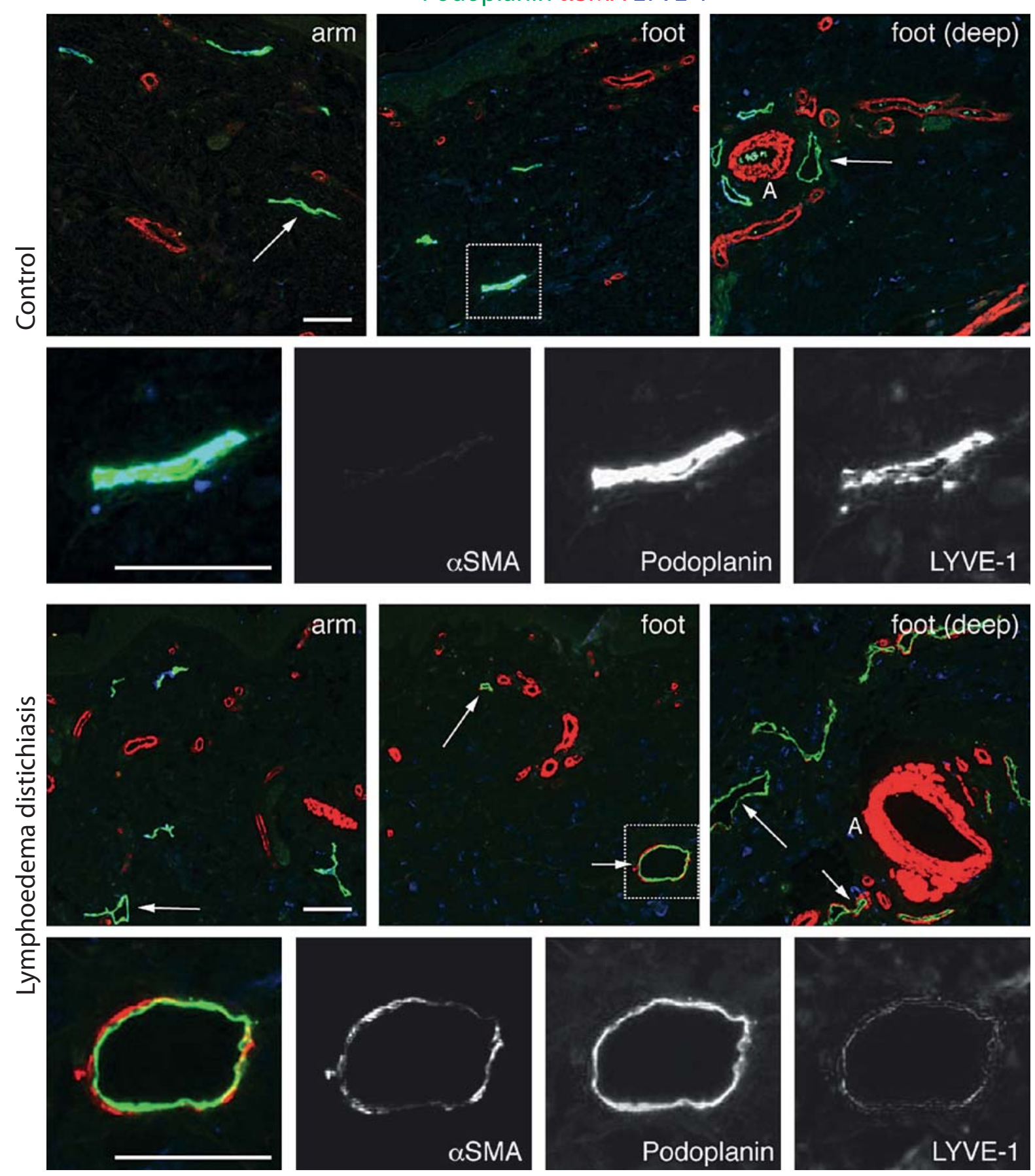

Fig. 5. Histological sections of skin biopsies from the ventral forearm and dorsal foot (the superficial and deep dermis are shown for the foot) of two participants: upper two rows, healthy control; lower two rows, lymphoedema distichiasis. Higher magnifications of the boxed areas in the foot sections are shown below. The sections illustrated were stained for podoplanin (green) and LYVE-1 (blue), as well as $\alpha$-SMA (red). Stained structures were only counted as lymphatics if they had a visible lumen (examples are indicated by long arrows). LYVE-1 immunohistochemistry is less selective than podoplanin for lymphatic vessels [24] and was not used for quantifying lymphatic densities, but the co-localisation of the two stains is evident. Some lymphatics in the foot of the lymphoedema distichiasis participants were associated with cells staining for $\alpha$-smooth muscle actin (indicated by short arrows). The podoplanin lymphatic densities were similar in the healthy control and lymphoedema distichiasis groups for each site, but densities were higher in the foot than in the forearm. A = Muscular arterioles. Scale bars $=100 \mu \mathrm{m}$. 
than arm. The forearm values observed by Stanton et al. [22] were a little higher than observed here, probably because a larger volume of injectate was used in the earlier study. The present results provide additional support for the 'greater transport capacity' concept; in healthy controls the lymphatic filling parameters in the foot were greater than in the forearm, and the biopsy results showed that the difference is at least partly due to a difference in microlymphatic density.

\section{Functional (Tracer-Filled) Lymphatic Density versus} Histological Lymphatic Density

As with the functional lymphatic density, the anatomical lymphatic vessel density determined by immunohistochemistry was higher in the foot than the forearm, by $31 \%$ in the control group and $39 \%$ in the FOXC2+/- group. The difference in histological density between foot and forearm was substantially smaller than the differences observed with FML (76 and 70\% for $\mathrm{LD}_{\max }$ in the control and LDS groups, respectively). This could be due to errors in vessel detection by either method, particularly immunohistochemistry, or it could be due to an influence of initial lymph formation rate (likely to be higher in the foot) on the number of vessels detected by FML.

\section{The Abnormal Lymphatic Response to Dependency} in LDS

In the foot of the penetrant FOXC2+/- subjects, but not the control or carrier groups, there was a significant reduction in lymphatic filling with tracer during dependency. This phenomenon has never previously been reported, to our knowledge, and it seems likely that the posture-dependent impairment is one of the primary factors causing the swelling of the interstitial compartment of the lower limb in LDS. The new finding raises the following question: By what mechanism might dependency adversely affect lymph uptake in LDS, but not in health?

The mutations in FOXC2 did not cause microlymphatic hypo-/aplasia, and did not influence forearm lymphatic filling or that of the LDS foot at heart level. These observations indicate that there is no intrinsic failure of the dermal microlymphatic ability to take up interstitial fluid, in contrast with the situation in humans with VEGFR3 mutations [24]. Moreover, oedema per se does not impair tracer uptake during FML, since uptake was normal in the swollen foot at heart level. Indeed, in breast cancer-related lymphoedema of the arm the number of tracer-filled microlymphatics is actually increased [20].

How might the deleterious effect of dependency on lymphatic filling in LDS subjects be explained? Although the present study does not establish the mechanism(s), three possibilities may merit future investigation.

(1) Failure of proximal lymphatic valves. There is clear evidence that the FOXC2 mutations impair semilunar valve competence in the leg veins of human LDS subjects [13] and impair semilunar valve formation in lymphatic trunk vessels of the mouse Foxc2 ${ }^{-/-}$model [12]. Clinical lymphoscintigraphy in LDS shows dermal backflow, which is interpreted as indicating lymph reflux, that is, retrograde lymph flow across a valve from collectors into dermal pre-collectors and initial lymphatics (although such flow cannot currently be imaged directly) [7].

(2) Abnormal investment of initial lymphatics with smooth muscle cells. An abnormal association of smooth muscle-like mural cells around the initial lymphatics of Foxc $2^{-/-}$mice was reported by Petrova et al. [12], an observation noted in the foot but not in the arm skin of the FOXC2+/- patients reported here (fig. 5). It is possible that these cells interfere with initial lymph absorption by contracting in response to dependency, in a manner analogous to the veni-arteriolar response of the microcirculation. In addition, ectopic smooth muscle cell coverage may lead to gene expression changes within lymphatic endothelia, thereby affecting vessel function. Interestingly, the expression of LYVE-1, a marker of initial lymphatic vessels, was reduced in the $\alpha$-SMA-positive lymphatic

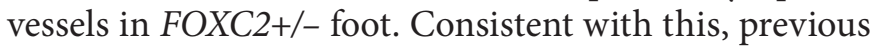
studies in mice reported that contact with smooth muscle cells regulates LYVE-1 expression in lymphatic endothelial cells [29].

(3) Venous distension. The gravitational pooling of refluxed blood in the upright posture, resulting from the multiple venous valve failure [13], might expand the leg veins sufficiently to compress adjacent collector lymphatics, leading to an increase in the pressure in distal (upstream) lymphatics, and in turn impairing effective fluid uptake [30, 31].

Taken in conjunction with the common embryological origin of the venous and lymphatic vessels, the above findings lead to the hypothesis that FOXC2 may be important in the normal development and maintenance of both lymphatic and venous luminal valves. Lymphatic semilunar valve failure seems to offer the most direct explanation for the FML finding that tracer uptake was only impaired only during orthostasis. When the lymphatic trunk vessels are in the vertical position, gravity acting on the vertical column of fluid will greatly raise the pressure in the microlymphatics of the foot unless competent semilunar valves break up the column of fluid into short segments, just as in leg veins. A large rise in lymph pres- 
sure inside the initial lymphatics $\left(\mathrm{P}_{\mathrm{L}}\right)$ during dependency will reverse the pressure gradient across the endothelial flap valve and thus close it [32]. Moreover the rise in $\mathrm{P}_{\mathrm{L}}$ will reduce or reverse the pressure gradient $\mathrm{P}_{\mathrm{i}}-\mathrm{P}_{\mathrm{L}}$ that drives interstitial fluid (pressure $P_{i}$ ) into the initial lymphatic. Thus, structural failure of the proximal semilunar valves will lead to functional failure of fluid uptake through the distal flap valves during dependency (effect of gravitational stress) but not at heart level (no gravitational stress). A key feature of this hypothesis, the posture-sensitive magnitude of $\mathrm{P}_{\mathrm{L}}$, should be testable in humans by using micropipettes to measure $\mathrm{P}_{\mathrm{L}}$ in the LDS foot at heart level and during dependency [33]

Since venous semilunar valves are undoubtedly also impaired in LDS it is likely that the oedema is of mixed origin, that is, it is caused by the combination of (1) increased microvascular fluid filtration, which results from dependent venous pressure elevation (averaged over time) when venous valves are incompetent, and (2) impaired uptake of microvascular filtrate into the initial lymphatic system, resulting from a functional failure of endothelial flap valves to open normally, which is secondary to excessive lymph pressure elevation during dependency when the proximal lymphatic semilunar valves are incompetent. In theory, therefore, overcoming the effects of gravity with compression therapy and elevation when resting from an early age in individuals with a FOXC2 mutation could prevent the onset of swelling, and in particular the secondary and irreversible changes that take place in untreated lymphoedema. Indeed, this has been observed in one of our other patients who has worn compression hosiery since teenage years.

\section{Conclusions}

This is the first study to our knowledge showing that fluid uptake into the initial lymphatic system of the lower limb in humans with FOXC2 mutations is impaired during dependency yet is normal at heart level. This phenomenon, whatever its mechanism, is likely to contribute importantly to the pathogenesis of the leg swelling in LDS. Indirect evidence raises the possibility that structural incompetence of the proximal semilunar valves in the lymph trunk vessels may be the cause of the functional failure of the initial lymphatic uptake process during gravitational stress (dependency). If the molecular mechanism for valve failure can be elucidated, it may provide a useful target for the development of treatment for LDS.

\section{Acknowledgements}

We thank the participants. We also thank G. Brice, C. Carver, A.H. Child, S. Mansour, P. Ostergaard, M. Sarfarazi and B. Sawyer (non-author members of the Lymphoedema Research Consortium) for recruitment of volunteers, determination of genotype and assistance with histological analysis; V. Akhras for assistance with histological analysis; the British Heart Foundation for funding this research (grant No. PG/02/111); the British Skin Foundation and Reece Spence Fund for further financial support. Institute where the research was conducted: St. George's, University of London, London, UK.

\section{References}

1 Bell R, Brice G, Child AH, Murday VA, Mansour S, Sandy CJ, Collin JR, Brady AF, Callen DF, Burnand K, Mortimer P, Jeffery S: Analysis of lymphoedema-distichiasis families for FOXC2 mutations reveals small insertions and deletions throughout the gene. Hum Genet 2001;108:546-551.

-2 Erickson RP, Dagenais SL, Caulder MS, Downs CA, Herman G, Jones MC, KerstjensFrederikse WS, Lidral AC, McDonald M, Nelson CC, Witte M, Glover TW: Clinical heterogeneity in lymphoedema-distichiasis with FOXC2 truncating mutations. J Med Genet 2001;38:761-766.
3 Fang J, Dagenais SL, Erickson RP, Arlt MF, Glynn MW, Gorski JL, Seaver LH, Glover TW: Mutations in FOXC2 (MFH-1), a forkhead family transcription factor, are responsible for the hereditary lymphedema-distichiasis syndrome. Am J Hum Genet 2000;67: 1382-1388.

-4 Finegold DN, Kimak MA, Lawrence EC, Levinson KL, Cherniske EM, Pober BR, Dunlap JW, Ferrell RE: Truncating mutations in FOXC2 cause multiple lymphedema syndromes. Hum Mol Genet 2001;10:11851189.
5 Kriederman BM, Myloyde TL, Witte MH, Dagenais SL, Witte CL, Rennels M, Bernas MJ, Lynch MT, Erickson RP, Caulder MS, Miura N, Jackson D, Brooks BP, Glover TW: FOXC2 haploinsufficient mice are a model for human autosomal dominant lymphedema-distichiasis syndrome. Hum Mol Genet 2003; 12:1179-1185.

6 Kume T, Jiang H, Topczewska JM, Hogan BL: The murine winged helix transcription factors, Foxc1 and Foxc2, are both required for cardiovascular development and somitogenesis. Genes Dev 2001;15:2470-2482. 
7 Brice G, Mansour S, Bell R, Collin JR, Child AH, Brady AF, Sarfarazi M, Burnand KG, Jeffery S, Mortimer P, Murday VA: Analysis of the phenotypic abnormalities in lymphoedema-distichiasis syndrome in 74 patients with FOXC2 mutations or linkage to $16 \mathrm{q} 24$. J Med Genet 2002;39:478-483.

-8 Connell F, Kalidas K, Ostergaard P, Brice G, Homfray T, Roberts L, Bunyan DJ, Mitton S, Mansour S, Mortimer P, Jeffery S, Lymphoedema Consortium: Linkage and sequence analysis indicate that CCBE1 is mutated in recessively inherited generalised lymphatic dysplasia. Hum Genet 2010;127:231-241. Erratum: Hum Genet 2010;127:243.

9 Irrthum A, Karkkainen MJ, Devriendt K, Alitalo K, Vikkula M: Congenital hereditary lymphedema caused by a mutation that inactivates VEGFR3 tyrosine kinase. Am J Hum Genet 2000;67:295-301.

-10 Irrthum A, Devriendt K, Chitayat D, Matthijs G, Glade C, Steijlen PM, Fryns JP, Van Steensel MA, Vikkula M: Mutations in the transcription factor gene SOX18 underlie recessive and dominant forms of hypotrichosis-lymphedema-telangiectasia. Am J Hum Genet 2003;72:1470-1478.

-11 Dale RF: Primary lymphoedema when found with distichiasis is of the type defined as bilateral hyperplasia by lymphography. J Med Genet 1987;24:170-171.

-12 Petrova TV, Karpanen T, Norrmén C, Mellor R, Tamakoshi T, Finegold D, Ferrell R, Kerjaschki, Mortimer P, Ylä-Herttuala S, Miura N, Alitalo K: Defective valves and abnormal mural cell recruitment underlie lymphatic vascular failure in lymphedema distichiasis. Nat Med 2004;10:974-981.

-13 Mellor RH, Brice G, Stanton AWB, French J, Smith A, Jeffery S, Levick JR, Burnand KG, Mortimer PS: Mutations in FOXC2 are strongly associated with primary valve failure in veins of the lower limb. Circulation 2007;115:1912-1920.
$>14$

Levick JR, Michel CC: The effects of position and skin temperature on the capillary pressures in the fingers and toes. J Physiol 1978; 274:97-109.

-15 Noddeland H, Omvik P, Lund-Johansen P, Ofstad J, Aukland K: Interstitial colloid osmotic and hydrostatic pressures in human subcutaneous tissue during early stages of heart failure. Clin Physiol 1984;4:283-297.

16 Engeset A, Olszewski W, Jaeger PM, Sokolowski J, Theodorsen L: Twenty-four hour variation in flow and composition of leg lymph in normal men. Acta Physiol Scand 1977;99:140-148.

17 Olszewski WL, Engeset A, Sokolowski J: Lymph flow and protein in the normal male leg during lying, getting up, and walking. Lymphology 1977;10:178-83.

18 Olszewski WL, Engeset A: Intrinsic contractility of prenodal lymph vessels and lymph flow in human leg. Am J Physiol 1980; 239:H775-H783.

19 Bollinger A, Jäger K, Sgier F, Seglias J: Fluorescence microlymphography. Circulation 1981;64:1195-1200.

20 Mellor RH, Stanton AWB, Azarbod P, Sherman MD, LevickJR, Mortimer PS: Enhanced cutaneous lymphatic network in the forearms of women with postmastectomy oedema. J Vasc Res 2000;37:501-512.

-21 Stanton AWB, Kadoo P, Mortimer PS, Levick JR: Quantification of the initial lymphatic network in normal human forearm skin using fluorescence microlymphography and stereological methods. Microvasc Res 1997; 54:156-163.

22 Stanton AWB, Patel HS, Levick JR, Mortimer PS: Increased dermal lymphatic density in the human leg compared with the forearm. Microvasc Res 1999;57:320-328.

23 Weibel ER: Stereological principles for morphometry in electron microscopic cytology. Int Rev Cytol 1969;26:235-302.

-24 Mellor RH, Hubert C, Stanton AWB, Tate N, Akhras V, Smith A, Burnand KG, Jeffery S, Mäkinen T, Levick JR, Mortimer PS: Lymphatic dysfunction, not aplasia, underlies Milroy disease. Microcirculation 2010;17: 281-296.
25 Weninger W, Partanen TA, BreitenederGeleff S, Mayer C, Kowalski H, Mildner M, Pammer J, Stürzl M, Kerjaschki D, Alitalo K, Tschachler E: Expression of vascular endothelial growth factor receptor-3 and podoplanin suggests a lymphatic endothelial cell origin of Kaposi's sarcoma tumor cells. Lab Invest 1999;79:243-251.

-26 Fukunaga M: Expression of D2-40 in lymphatic endothelium of normal tissues and in vascular tumours. Histopathology 2005;46: 396-402.

27 Mortimer PS, Levick JR: Chronic peripheral oedema: the critical role of the lymphatic system. Clin Med 2004;4:448-453.

$>28$ Noddeland H: Influence of body posture on transcapillary pressures in human subcutaneous tissue. Scand J Clin Lab Invest 1982; 42:131-138.

-29 Tammela T, Saaristo A, Holopainen T, Lyytikkä J, Kotronen A, Pitkonen M, Abo-Ramadan U, Ylä-Herttuala S, Petrova TV, Alitalo K: Therapeutic differentiation and maturation of lymphatic vessels after lymph node dissection and transplantation. Nat Med 2007; 13:1458-1466.

30 Aukland K, Reed RK: Interstitial-lymphatic mechanisms in the control of extracellular fluid volume. Physiol Rev 1993;73:1-78.

31 Chen HI, Granger HJ, Taylor AE: Lymph flow transient following elevation of venous pressure in the dog's hind-paw; in Mayall RC, Witte MH (eds): Progress in Lymphology. New York, Plenum, 1977, pp 19-26.

32 Schmid-Schönbein GW: Microlymphatics and lymph flow. Physiol Rev 1990;70:987-

33 Franzeck UK, Fischer M, Costanzo U, Herrig I, Bollinger A: Effect of postural changes on human lymphatic capillary pressure of the skin. J Physiol 1996;494:595-600. 